\title{
"El rol de la ingeniería y de la investigación en ingeniería en el proceso de licenciamiento ambiental"
}

\author{
“The role of Engineering and Engineering Science in Environmental Licensing”
}

\author{
Carlos Costa Posada ${ }^{(1)}$ \\ Editor invitado \\ (1) Exministro de Ambiente, Vivienda y Desarrollo. Decano, Facultad de Ingeniería, Universidad de la Salle, Bogotá D.C., Colombia. \\ crcosta@lasalle.edu.co
}

No es necesario explicar que el desarrollo del país debe hacerse de manera sostenible, pero la pregunta muchas veces es ¿cómo hacerlo? Y en ese cómo hacerlo uno empieza a mirar y se da cuenta de algo que es obvio, pero que no es claro para todo el mundo: la ingeniería está metida en todo el proceso de gestión ambiental, desde los proyectos que impactan el medio ambiente, hasta las soluciones que se plantean para mitigar o controlar esos impactos pasando por los temas de regulación ambiental, de monitoreo, de modelación y de seguimiento. Claramente se ve que este es un tema muy de la ingeniería y es ella quien tiene que atender buena parte de las respuestas a esa pregunta.

Con todo lo anterior en mente, decidimos organizar un foro cuyo tema central fuera qué puede hacer la ingeniería y la investigación en ingeniería para aportarle al proceso de licenciamiento ambiental, de monitoreo y de seguimiento. Si la ingeniería está metida en todo esto, no podemos seguir quejándonos, más bien, buscar la manera de meternos en el asunto y aportar soluciones. Eso fue lo que quisimos hacer. De ahí que hayamos invitado a personas de distintos sectores para tener una visión integral del tema. En primer lugar, está el Dr. Pablo Vieira, viceministro de Ambiente y Desarrollo Sostenible, quién hablará de su visión como ingeniero $y$, también, de cómo se ve el tema desde el Gobierno. Cabe aclarar que en este foro buscamos una conversación con personas y profesionales, más que con instituciones propiamente dichas. En segundo lugar, está el Dr. Barron Henderson, profesor de la Universidad de Florida, quien ha trabajado con la Environmental Protection Agency (EPA) y está detrás del respaldo conceptual de algunas de las regulaciones ambientales en Estados Unidos. Su fortaleza es el tema de modelación de la calidad del aire. En tercer lugar, tenemos al Dr. Ignacio Toro, quien tiene una trayectoria muy importante en el sector ambiental en Chile. Él ha sido Es director ejecutivo de la Comisión Nacional del Medio Ambiente (CONAMA) y Es director ejecutivo del Servicio de Evaluación Ambiental (SEA). La concepción de un servicio de información ambiental es clave en esta tarea.

Más adelante, veremos la visión de la Dra. Ángela Cadena, profesora de la Universidad de los Andes, quien también ha ocupado cargos públicos importantes relacionados con el desarrollo de la infraestructura en el país. Ella representa a la academia y mostrará la oferta de programas y grupos de investigación en temas ambientales que hay en las universidades del país y los retos que desde allí se plantean para mejorar. Por otra parte, el Dr. Tirso Quintero nos dará su mirada desde el sector privado. Él es el gerente de Consultores Unidos S.A. La consultoría en ingeniería en Colombia juega un rol fundamental en todo el proceso de licenciamiento ambiental. La mayoría de las empresas que van a buscar una licencia ambiental no tienen la capacidad instalada

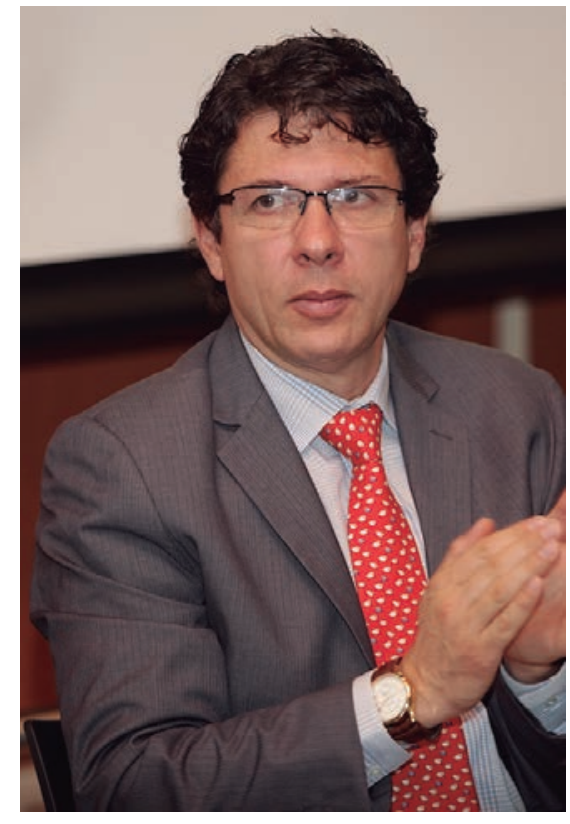

Carlos Costa Posada. Fuente: Archivo Particular, Universidad de los Andes

para estar en un proceso de solicitud de licenciamiento ambiental y, por eso, se apoyan en la consultoría. Finalmente, están la Dra. Elsa Matilde Escobar y el Dr. Roberto Gómez en representación de la sociedad civil. Escobar es directora de la Fundación Natura, la ONG ambiental más seria y con mayor trayectoria, reconocimiento y visibilidad en el país. Ellos nos contarán sobre cómo ven desde su organización y su equipo el rol de la ingeniería en el proceso de licenciamiento ambiental.

A este grupo de personas, que representan distintas áreas involucradas en estos procesos, les pedimos que pensaran cómo creían que la ingeniería y la 
investigación en ingeniería podían contribuir a mejorar el proceso de licenciamiento ambiental y de seguimiento al licenciamiento. Muchas veces se nos olvida que el licenciamiento es apenas la primera parte, pero después, uno tiene que encargarse de hacer seguimiento a ver si lo que uno predijo se está comportando como se predijo y, si no se está comportando como se predijo, hay que intervenir inmediatamente y modificar las cosas. Aquí queremos darle un balance a esas dos partes: licenciamiento ambiental y seguimiento a ese proceso.

Los objetivos que propusimos para guiar la discusión en torno al tema fueron por un lado, cómo hacer para generar confianza entre las partes y reducir la incertidumbre. El licenciamiento ambiental se otorga bajo modelos pero no hay nada construido a la hora de tomar decisiones. Por otra parte, pensar en cómo estandarizar el proceso de licenciamiento y de seguimiento para generar reglas claras para todos.

La intención de este evento que era ver cómo la ingeniería puede contribuir en el proceso de licenciamiento ambiental, sin negar que haya otras disciplinas que puedan hacerlo y que la interdisciplinariedad es importantísima para resolver los problemas. Sin embargo, en esa respuesta de que todos somos responsables de todo, nadie termina siendo responsable de nada y se diluye toda responsabilidad e interés. Por esta razón, queríamos tener una discusión enfocada en el tema de la ingeniería para entender la situación y generar una agenda de trabajo.

Las principales conclusiones de las ponencias que se presentaron durante el foro fueron:

- La participación de la ingeniería y de la academia le da mucha credibilidad al proceso y le da mucha confianza a las partes. Hay un llamado general a que la ingeniería se involucre en el espacio de conceptualización, en esa primera gran etapa del diseño normativo y de los procedimientos; hay un llamado de todos los panelistas a la necesidad de estandarizar el proceso de licenciamiento ambiental. Muchas veces esos procesos, las normas y la estandarización están en manos de los funcionarios de turno y de las autoridades, y no en manos de quienes le podrían dar más credibilidad como son la academia o los expertos. Ahí hay un primer espacio de entrada a la ingeniería para que participe en esos procesos de diseño metodológico y de diseño normativo de la mano con la autoridad también, pero que haya un tercero neutral. Ese llamado que nos hacía el profesor Henderson de que la ciencia y la academia finalmente están expuestas a una revisión de pares y hay una sensación de neutralidad. Ese proceso tiene que ser muy participativo. No se trata de contratar a una universidad para que se encargue de hacer la propuesta, sino que haya un proceso abierto donde participe todo el sector académico, y no simplemente aquellos que fueron contratados. Es necesario que haya un espacio para que la sociedad civil y la empresa privada participen. Yo creo que ese es el primer reto que sale de este foro: ¿cómo hacemos para que esa participación de la academia en los procesos normativos sea constante 0 , sea de alguna manera permanente? La mayoría de las veces se contrata a alguien para que haga una parte, luego a otro y a otro y eso no genera la estabilidad que queremos. ¿Cómo generamos un proceso en donde la academia pueda participar de manera permanente, para que se dé espacio a los temas de participación, a los temas de apertura y que no sean cosas muy puntuales?

- La segunda área donde la ingeniería y la academia pueden jugar un rol importante es en el tema de la modelación y de la información. Para tomar decisiones, para reducir la incertidumbre es clave tener modelos que nos permitan hacer previsión de lo que va a ocurrir en el futuro, hacer pronósticos y se necesita información clave. Hay muchas entidades recogiendo información pero, como decía la Dra. Escobar, muchas veces no está disponible oportunamente la información. Hay mucha información sobre unas cosas pero sobre otras no. Cuando uno ve el sistema de información ambiental piensa que es maravilloso porque hay cinco institutos de investigación, todos recogiendo información sobre ecosistemas, biodiversidad, recurso hídrico, recurso forestal, entre otros. Sin embargo, cuando uno va a tratar de hacer procesos de estudio de impacto ambiental resulta que esa información sobre los recursos naturales sí es importante, pero también necesito información sobre los demás proyectos que están licenciados en la zona, sobre el estado actual de contaminación del espacio en donde voy a trabajar y no hay una estrategia -aunque en este momento el ANLA está empezando a tratar de montarla-, que le diga a uno cuáles son los proyectos que están licenciados y la información asociada a esos proyectos tampoco está estandarizada. No es fácil sumar este proyecto con el otro, conocer el grado de la contaminación ambiental que produce este proyecto porque el indicador que se usó de contaminación ambiental para este proyecto en materia de calidad de agua es diferente a la del otro; se usaron tiempos distintos o estándares diferentes y no son sumables. Este es un punto también importante de cómo puede la academia ayudar a definir cuáles son los modelos con los que se debe modelar o con los que hay una incertidumbre manejable, cuáles son los indicadores que se deben usar de manera que haya también una unidad de criterio.

- El tercer elemento es el tema de acompañar al sector privado a hacer los estudios de impacto ambiental. Eso es algo que está en 
manos de los ingenieros y de las consultorías en ingeniería en este momento. Ahí yo creo que la recomendación que hemos escuchado de todos es que necesitamos como universidades y como ingeniería en general asegurarnos de que estamos formando a los mejores ingenieros posibles porque ellos son los que van a estar detrás de estos procesos y ahí es donde necesitamos trabajar el tema de la multidisciplinariedad, de la apertura a otras formas de ver las cosas, a generar esa sensibilidad por los temas ambientales en los ingenieros en su proceso de formación desde temprano. Es el tema realmente en que nosotros como ingeniería, como instituciones, como universidades deberíamos estar involucrados con fuerza.

- La etapa final de esto, luego de acompañar a los procesos de estudios de impacto, es la de monitoreo y seguimiento de proyectos licenciados. Yo creo que ahí también la ingeniería y la academia tienen un rol muy importante. Como lo planteó la Dra. Escobar, sería interesante que hubiera un monitoreo comunitario, que tendría un rol muy importante y también podría estar en manos de la academia y de la comunidad. Le daría muchísima credibilidad al proceso que hubiera un sistema en donde el monitoreo del seguimiento en la ejecución de los planes de manejo ambiental, de algunas variables por lo menos, $\mathrm{y}$ del impacto ambiental del proyecto estuvieran en manos de un consorcio academia-comunidad.

- Si nosotros logramos estandarizar el proceso de licenciamiento ambiental, luego de un proceso transparente que le de credibilidad a las partes y no simplemente una adopción de una norma por parte de una entidad en particular, sino un proceso estándar que es el resultado de un proceso de participación de personas creíbles, de entidades confiables, estandarizamos ese proceso pero también estandarizamos o simplificamos u orientamos el tema de las normas, los modelos a usar, las variables que se van a utilizar, eso puede que haga más complejo el proceso de la evaluación de impacto ambiental pero debe simplificar la evaluación por parte del evaluador, debe reducir la incertidumbre de los plazos, de los tiempos, y el resultado final es tener más tiempo disponible para dedicarnos a lo importante: la evaluación de los impactos ambientales, la evaluación de las alternativas de mitigación, la evaluación del seguimiento del proyecto y del plan de manejo ambiental que es lo que le daría valor agregado al proceso. Lo que llamaba a la mesa la Dra. Escobar ¿cómo hacemos para que este proceso le de valor agregado, que no sea simplemente un requisito, sino que le de valor agregado? Ese podernos dedicar durante todo este tiempo a hacer cosas que le aporten valor agregado sin tener que pasar mucho tiempo revisando que si tienen los elementos apropiados, si el modelo es confiable, si se están cumpliendo con todos los elementos de los manuales, en fin, mucho tiempo revisando cosas muy rutinarias y cuando ya se pasó el tiempo decir "toca pasar la licencia porque llevamos 300 días por encima del tiempo"; nos toca dar la licencia ambiental y nunca tuvimos el tiempo que hubiéramos querido para sentarnos a mirar las alternativas de manejo del proyecto. Nos mostraban en Chile un ejemplo muy interesante de cómo el proyecto concluye que la mejor decisión es invertir en otro proyecto para reducir la contaminación y no tener que hacerlo todo uno aquí, pero ese tipo de aportes solamente salen cuando todos los que estamos involucrados en el proceso de licenciamiento ambiental tenemos tiempo para pensar en la parte propositiva, sin gastarnos todo el tiempo en la parte normativa, cumple, está hecho de manera rigurosa, el modelo es confiable, la variable, está considerando los vecinos. No, todo eso debería estar lo suficientemente estandarizado en el proceso para que los evaluadores se pudieran dedicar a mirar lo realmente importante que es cómo se le puede dar valor agregado y si efectivamente se están considerando las mejores alternativas para minimizar el impacto ambiental y maximizar los resultados positivos. 